\title{
Innate Sulfur Compounds as Internal Standard for Determining Vacuum Gas Oil Compositions by APPI FT-ICR MS
}

\author{
Hendrik Muller*, Nadrah A. Alawani, Frederick M. Adam \\ Saudi Aramco, Research \& Development Center, Dhahran, 31311, Saudi Arabia
}

\section{Supporting Information}

In this work, innate sulfur components in high boiling petroleum samples were used as internal standard for the estimation of the entire sample composition through atmospheric pressure photo ionization (APPI) Fourier-transform ion cyclotron resonance mass spectrometry (FT-ICR MS). In a first step a maximally representative mass spectrum was recorded for the aromatic components, using time-of-flight mass spectrometry (TOF MS) to tune the FT-ICR MS parameters. Then, the identification of individual ion species and their mass spectrometric abundance was combined with the total sulfur content information to obtain the mass fraction of aromatic sulfur species present. Aromatic hydrocarbon (HC) species' quantification was based on equimolar response compared to aromatic sulfur species. Nitrogen species were treated analogously to sulfur compounds by using the total nitrogen content. The mass balance of all aromatic compounds yields the mass fraction of saturated compounds, which are not directly accessible with the APPI process.

To assess the validity and biases of this mass spectrometry approach, comprehensive 2dimensional gas chromatography was used as a reference technique, and the saturated compound fractions were determined gravimetrically, and also characterized separately using field desorption/field ionization TOF MS to yield additional compositional information. The analytical approach was evaluated on a set vacuum distilled fractions with narrow boiling ranges, obtained from Arabian Light crude oil. The method was then applied to two typical vacuum gas oil samples (obtained from a Norwegian and an Arabian crude oil) to derive detailed compositional information. 


\section{Table of Contents}

- Comparison of mass spectra by TOF-MS and FT-ICR MS for three VGO cut samples (Figure SI1)

- Average composition and standard deviation as error bars (Figure SI2)

- Relative expanded uncertainty of the APPI FT-ICR MS method results based on mass fraction per aromatic ring family (Figure SI3)

- Qualitative APPI FT-ICR MS results for VGO Cut\#14, including mass spectrum, class distribution, and carbon number vs DBE plots of the main classes (Figure S4I)

- 2-dimensional gas chromatogram of Cut \#14 with aromatic ring family identifications (Figure SI5)

- 2-dimensional chromatogram for Cut \#14 with ring number families indicated (Figure SI6)

- Sulfur speciation results for Cut \#14 with carbon number and molecular weight details based on identification by GCxGC-SCD (Table SI1)

- Carbon number distributions for benzothiophene and triaroamtic sulfur compound families resolved by GCxGC-SCD and APPI FT-ICR MS (Figure SI7)

- Qualitative APPI FT-ICR MS results for typical wide boiling range VGO samples, including mass spectrum, class distribution, and carbon number vs DBE plots of the main classes (Figure SI8)

- SATs content for VGO AL sample by SARA fractionation and the proposed APPI FT-ICR MS method (Figure SI9)

- SATs speciation by FD TOF MS mass spectrum and carbon number vs. DBE plot of saturated hydrocarbon species, where DBE directly reflects the number of naphthenic rings (Figure SI10) 


\section{APPI TOF-MS}

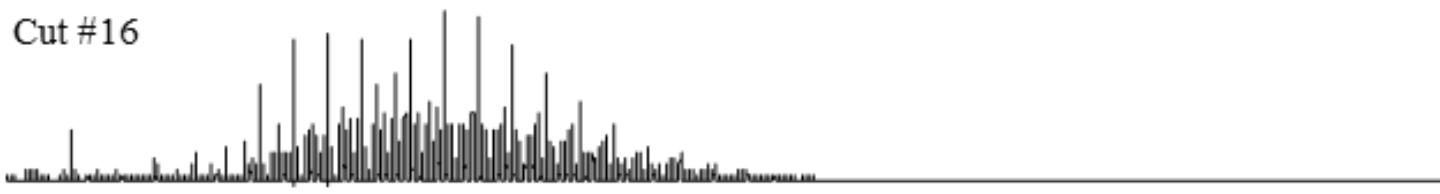

Cut \#20

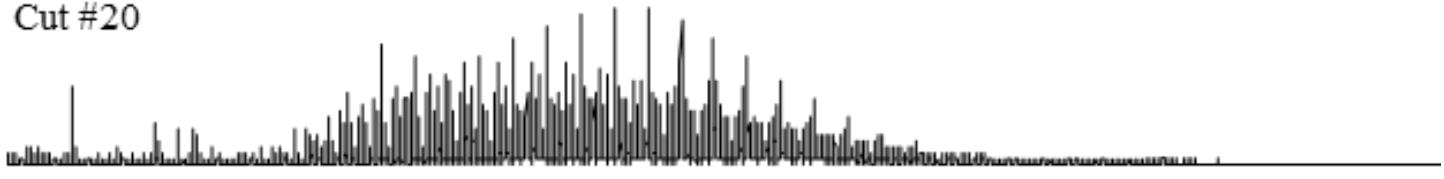

Cut \#23

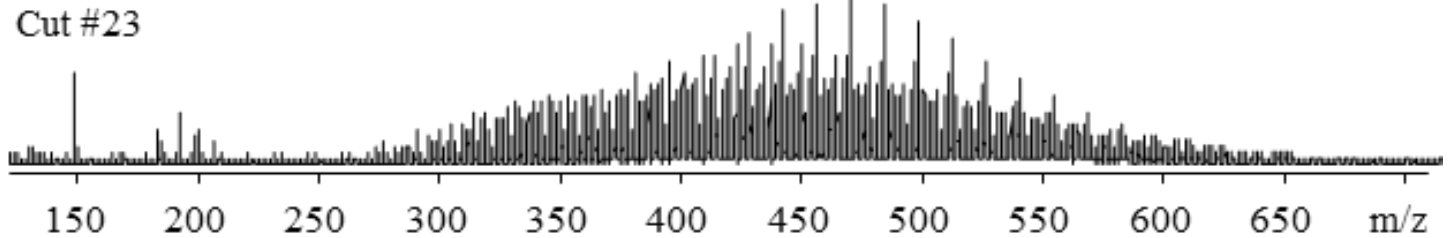

\section{APPI FT-ICR-MS}
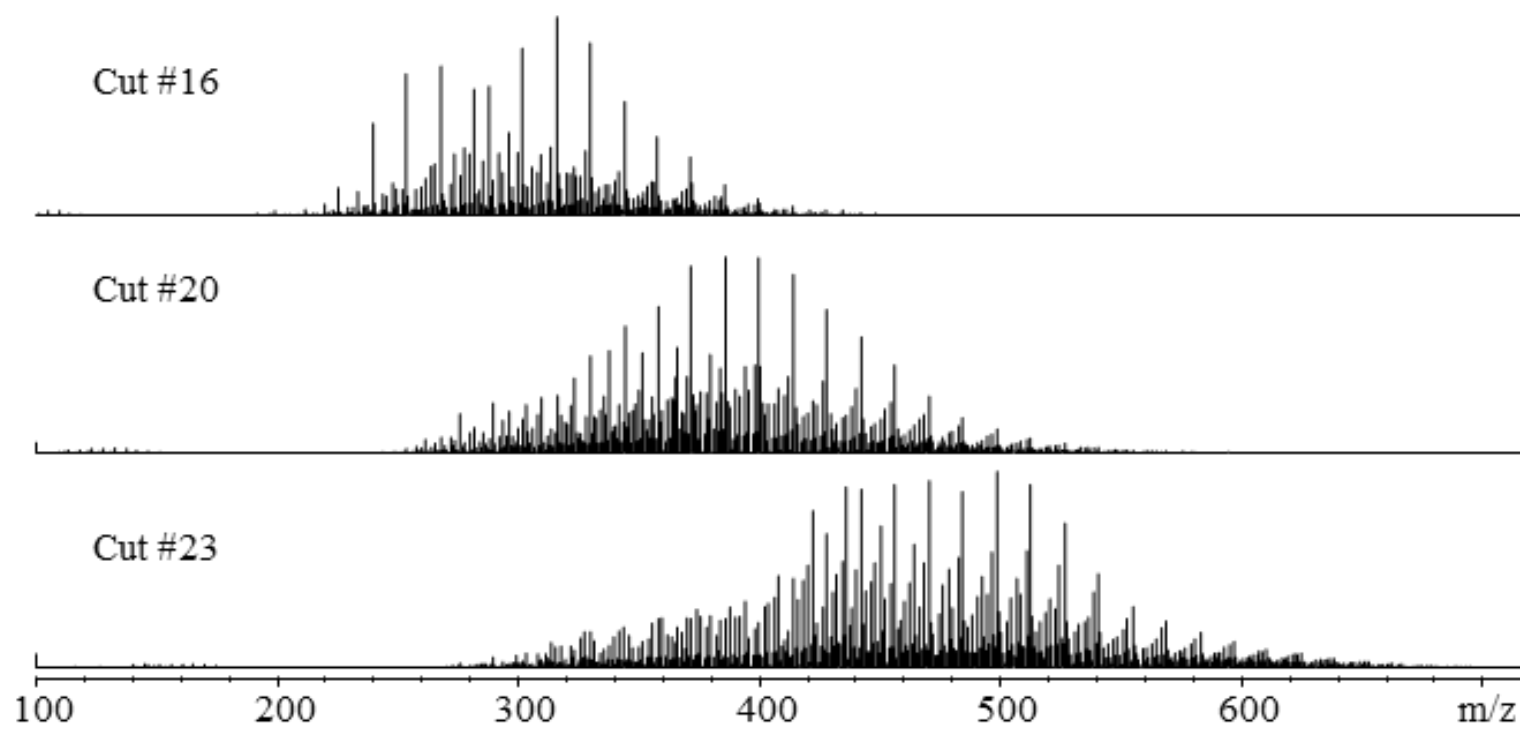

Figure SI1. APPI mass spectra obtained using TOF and FT-ICR mass analyzers. The spectra match $\mathrm{m} / \mathrm{z}$ signal distributions after tuning the FT-ICR MS ion transmission parameters. 


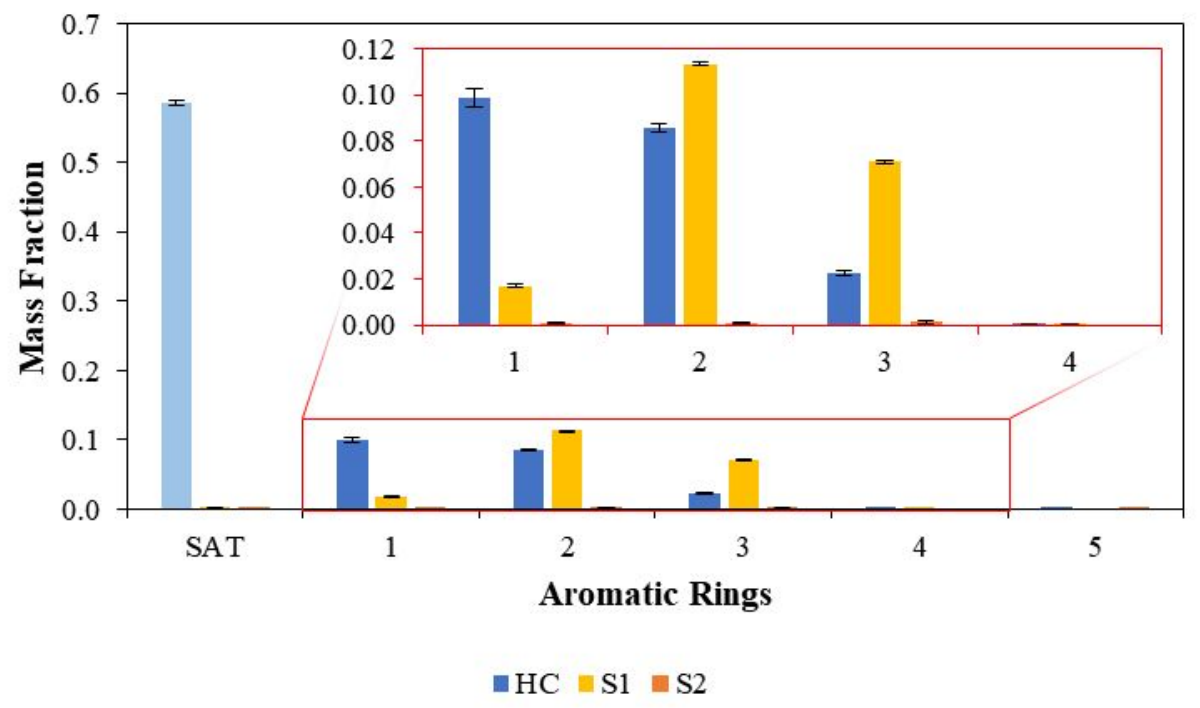

Figure SI2. Repeatability of the MS method for Cut \#15; average results and standard deviations (error bars) are shown for 8 repeat measurements. The expanded region shows the details for aromatic families.

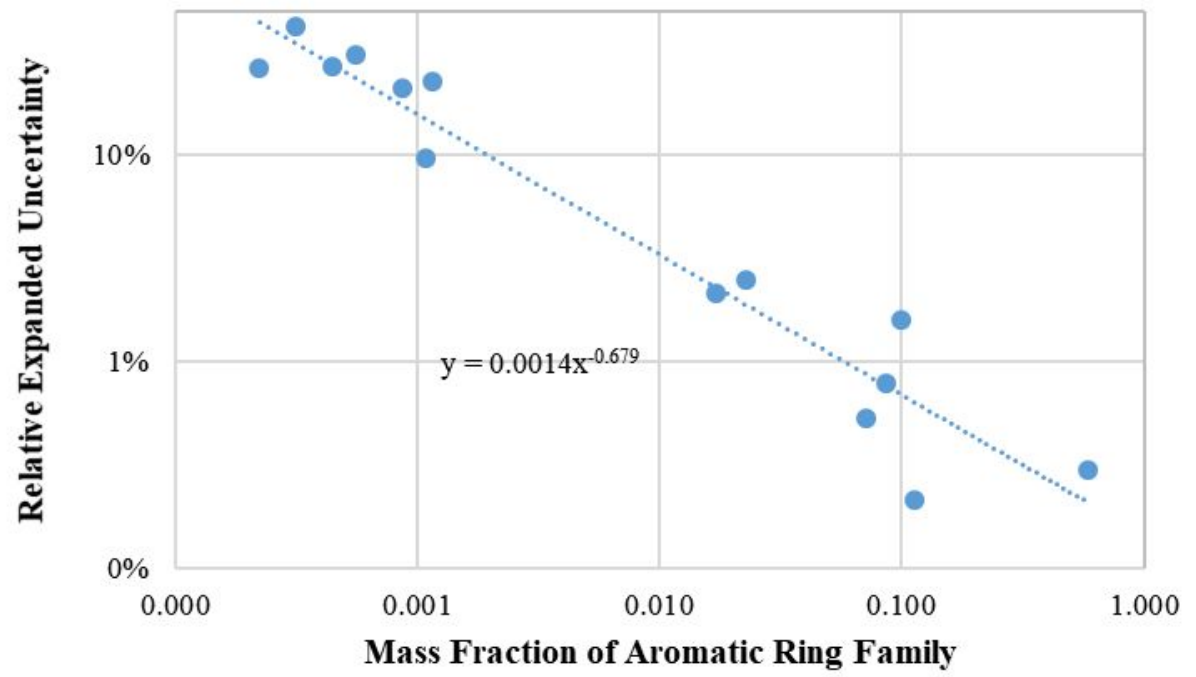

Figure SI3. Relative expanded uncertainty of aromatic ring families obtained for 8 repeat measurements using the presented MS method. For aromatic ring families with an estimated mass faction above 0.025 the relative expanded uncertainty is below $2.5 \%$. The uncertainty of the estimated mass fraction increases for less abundant aromatic ring families, as expected. Both axes are plotted in a logarithmic scale. 

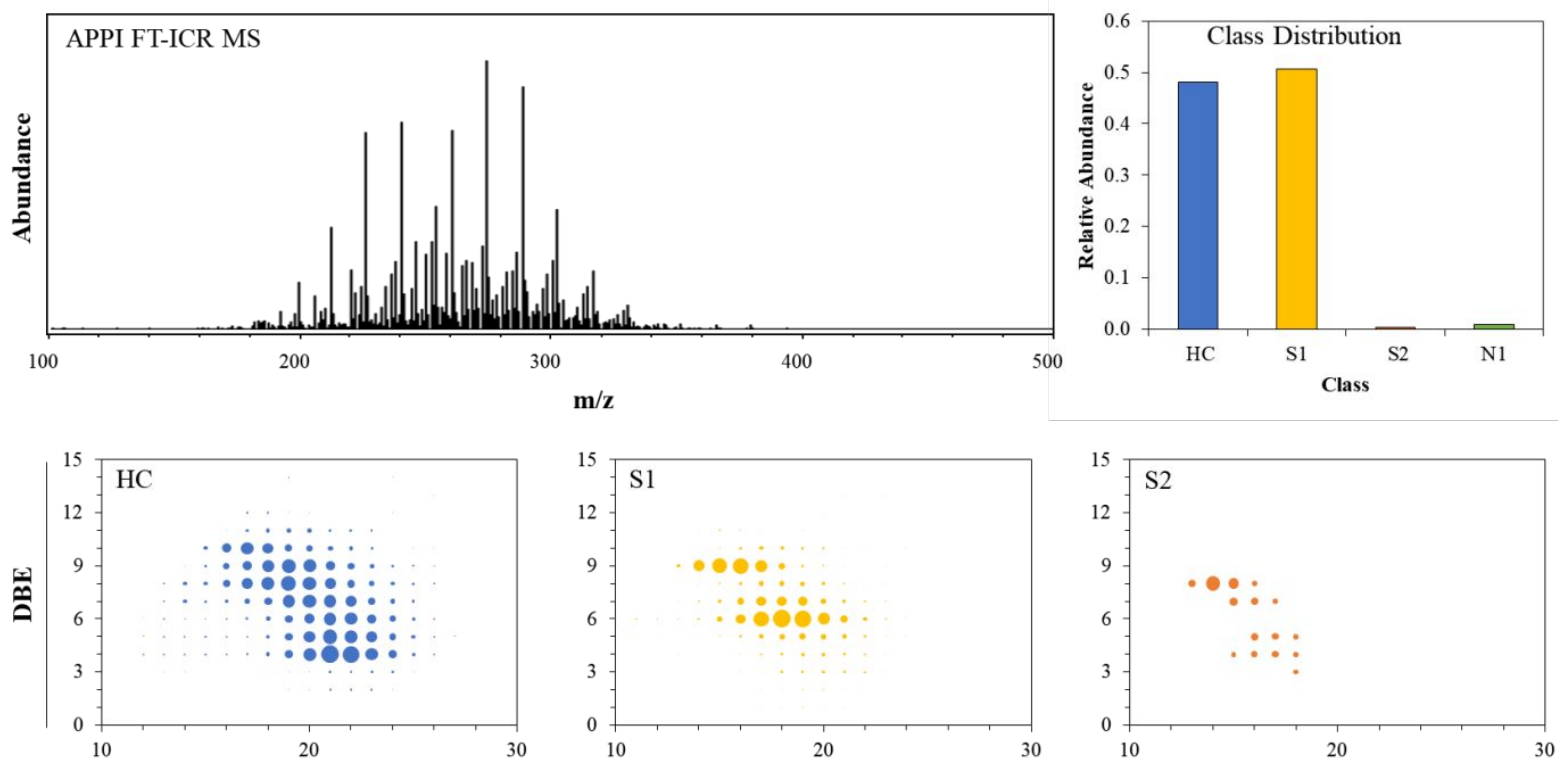

Carbon Number

Figure SI4. Mass spectrometry data for VGO Cut \#14, top: APPI FT-ICR mass spectrum and class distribution, bottom: carbon number vs DBE plots for $\mathrm{HC}, \mathrm{S} 1$, and S2 class species, respectively, where the dots' area represents their mass spectral abundance. 


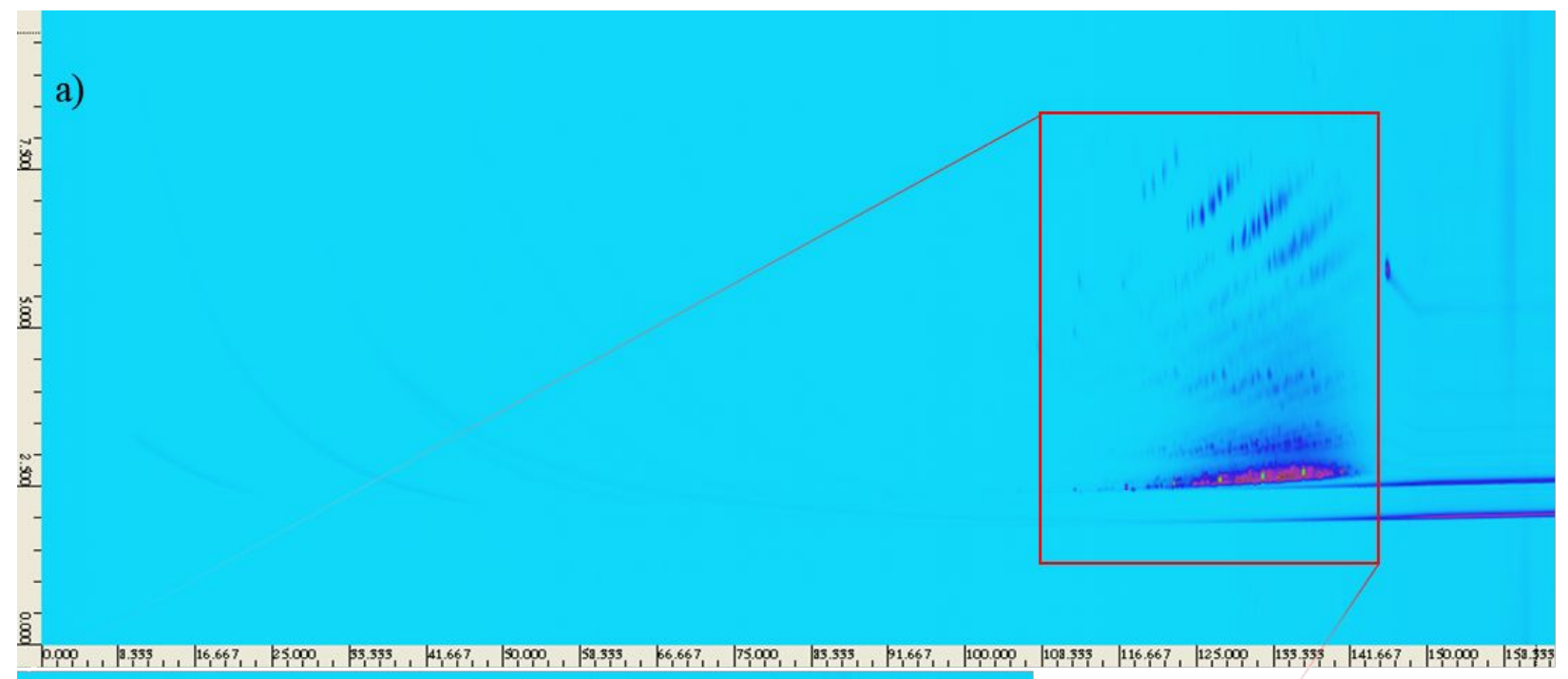

b)

c)

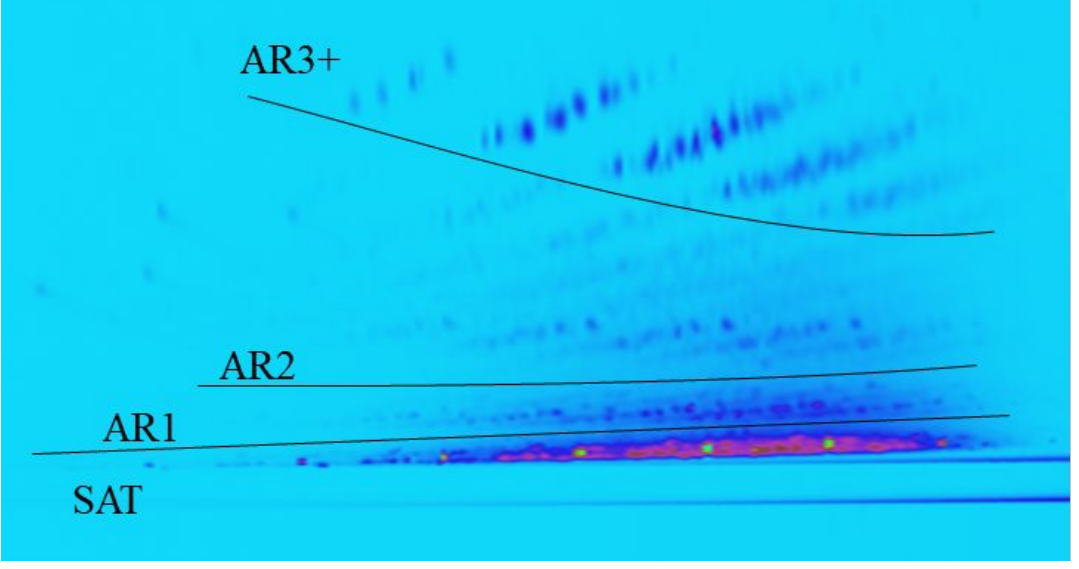

\begin{tabular}{|c|c|}
\hline $\begin{array}{c}\text { Compound } \\
\text { Family }\end{array}$ & $\begin{array}{c}\text { FID rel. } \\
\text { response }\end{array}$ \\
\hline SAT & $61.4 \%$ \\
\hline AR1 & $15.3 \%$ \\
\hline AR2 & $13.3 \%$ \\
\hline AR3+ & $10.1 \%$ \\
\hline
\end{tabular}

Figure SI5. a) 2-dimensional gas chromatogram of a light vacuum gas oil distillate (Cut \#14); b) zoomed chromatogram of the $1^{\text {st }}$ dimension between $105 \mathrm{~min}$ and $142 \mathrm{~min}$, and c) aromatic ring family distribution. 


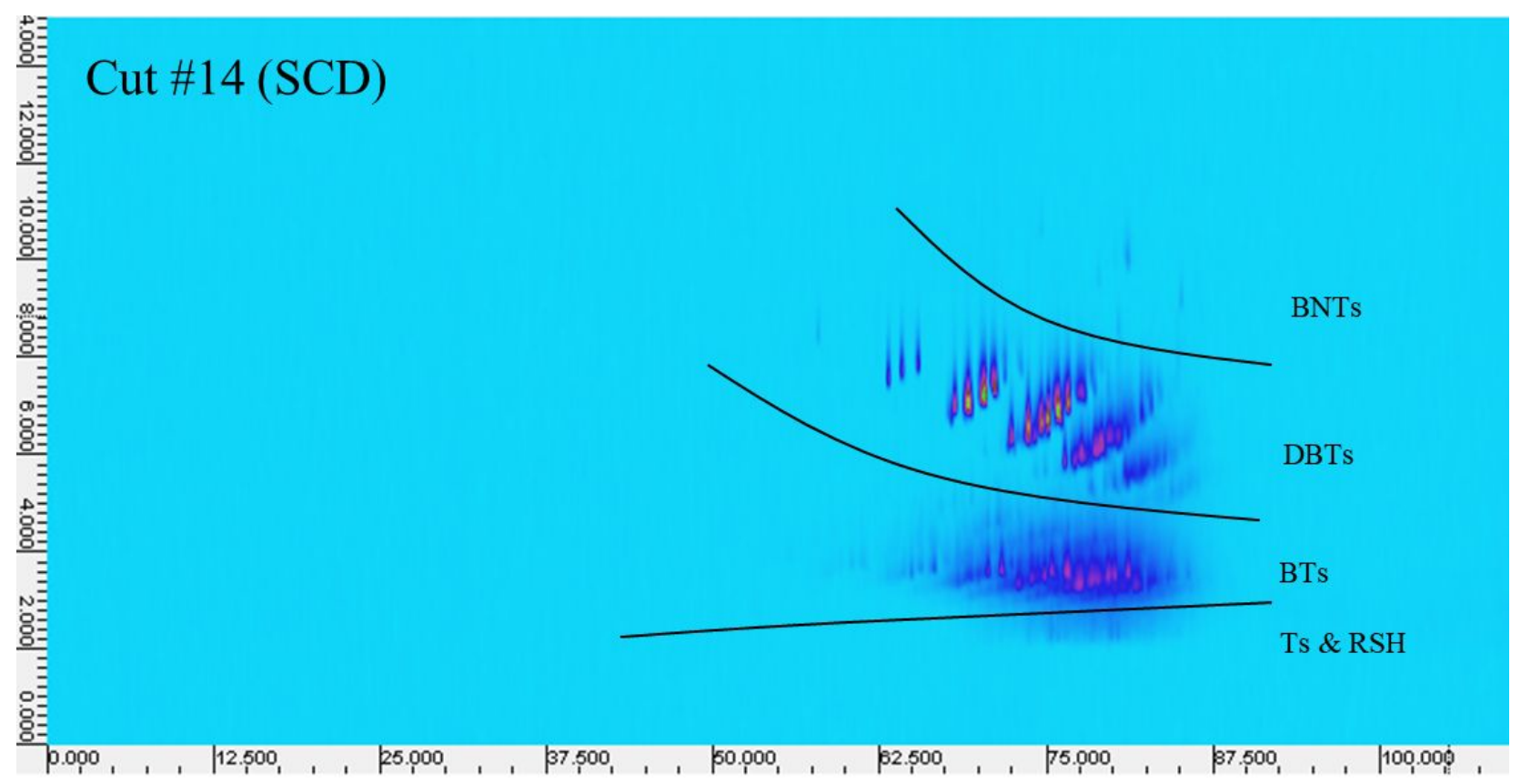

Figure SI6. Sulfur selective 2-dimensional gas chromatogram obtained by GCxGC SCD for Cut \#14. Thiophenes (Ts) and sulfides (RSH), diaromatic sulfur (namely benzothiophenes, BTs), triaromatic sulfur compounds (mainly dibenzothiophenes, DBTs), and few tetra-aromatic sulfur compounds (tentatively labeled as benzonaphthothiophenes, BNTs) are indicated. A separation by carbon atom number was achieved for the aromatic ring families. 
Table SI1. GCxGC-SCD speciation results for Cut \#14, with carbon number (C\#) and molecular weight (MW) based on the identification.

\begin{tabular}{|c|c|c|c|}
\hline Compound & SCD & $\mathrm{C \#}$ & MW \\
\hline $\mathrm{T}+\mathrm{RSH}$ & $8.14 \%$ & & \\
\hline BT_C04 & $0.75 \%$ & 12 & 190.0816 \\
\hline BT_C05 & $0.61 \%$ & 13 & 204.0972 \\
\hline BT_C06 & $0.72 \%$ & 14 & 218.1129 \\
\hline BT_C07 & $2.04 \%$ & 15 & 232.1285 \\
\hline BT_C08 & $3.42 \%$ & 16 & 246.1442 \\
\hline BT_C09 & $7.11 \%$ & 17 & 260.1598 \\
\hline BT_C10 & $8.05 \%$ & 18 & 274.1755 \\
\hline BT_C11 & $5.25 \%$ & 19 & 288.1911 \\
\hline BT_C12 & $1.41 \%$ & 20 & 302.2068 \\
\hline BT_C13 & $0.41 \%$ & 21 & 316.2224 \\
\hline BT_C14 & $0.18 \%$ & 22 & 330.2381 \\
\hline NT & $14.02 \%$ & 12 & 175.972 \\
\hline DBT_C00 & $0.42 \%$ & 12 & 184.0346 \\
\hline DBT_C01 & $2.05 \%$ & 13 & 198.0503 \\
\hline DBT_C02 & $10.33 \%$ & 14 & 212.0659 \\
\hline DBT_C03 & $15.65 \%$ & 15 & 226.0816 \\
\hline DBT_C04 & $11.83 \%$ & 16 & 240.0972 \\
\hline DBT_C05 & $4.93 \%$ & 17 & 254.1129 \\
\hline DBT_C06 & $1.41 \%$ & 18 & 268.1285 \\
\hline DBT_C07 & $0.36 \%$ & 19 & 282.1442 \\
\hline DBT_C08 & $0.24 \%$ & 20 & 296.1598 \\
\hline BNT_C00 & $0.30 \%$ & 16 & 234.0503 \\
\hline BNT_C01 & $0.38 \%$ & 17 & 248.0659 \\
\hline
\end{tabular}




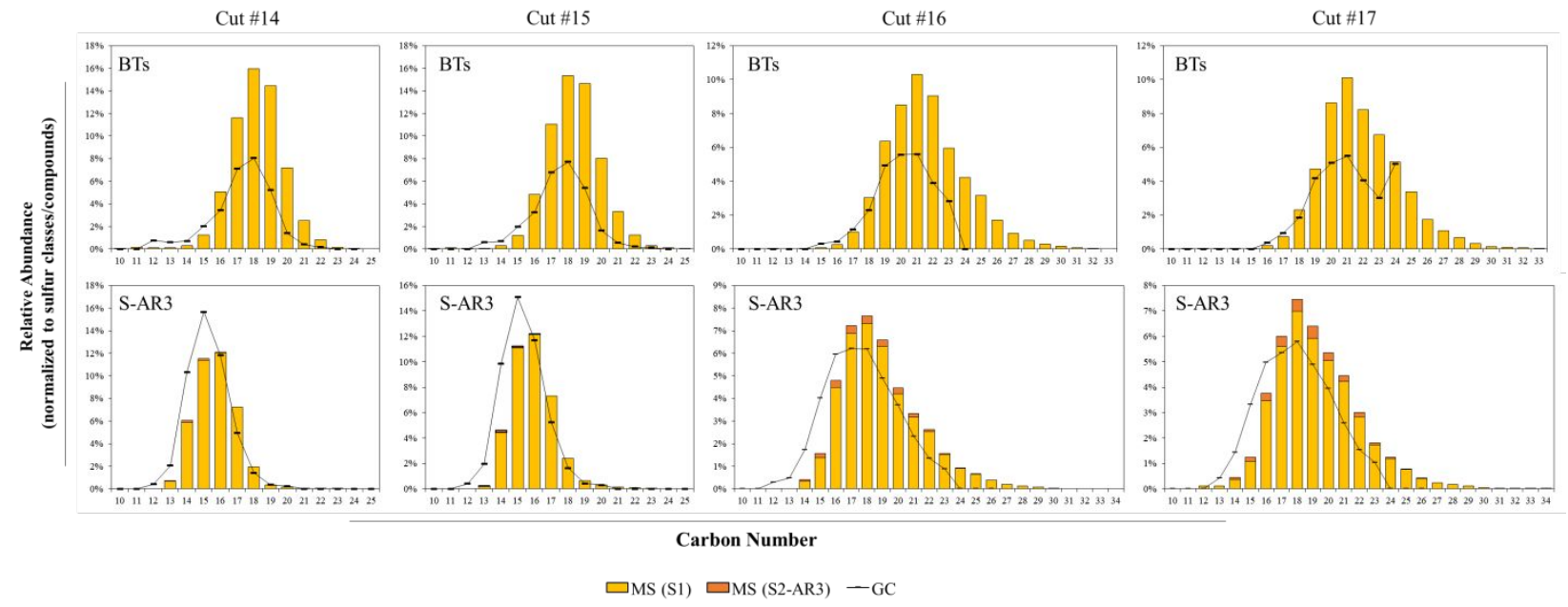

Figure SI7. Carbon number distributions for sulfur compounds by GCxGC-SCD speciation results (line, GC) and APPI FT-ICR MS (bars, MS). The top row shows data for benzothiophenes (BTs) and the bottom row triaromatic sulfur compounds (S-AR3) including S1 and S2 class species. The y-axes reflect the measurement abundance normalized to all sulfur containing species in the sample. 
a) VGO AL
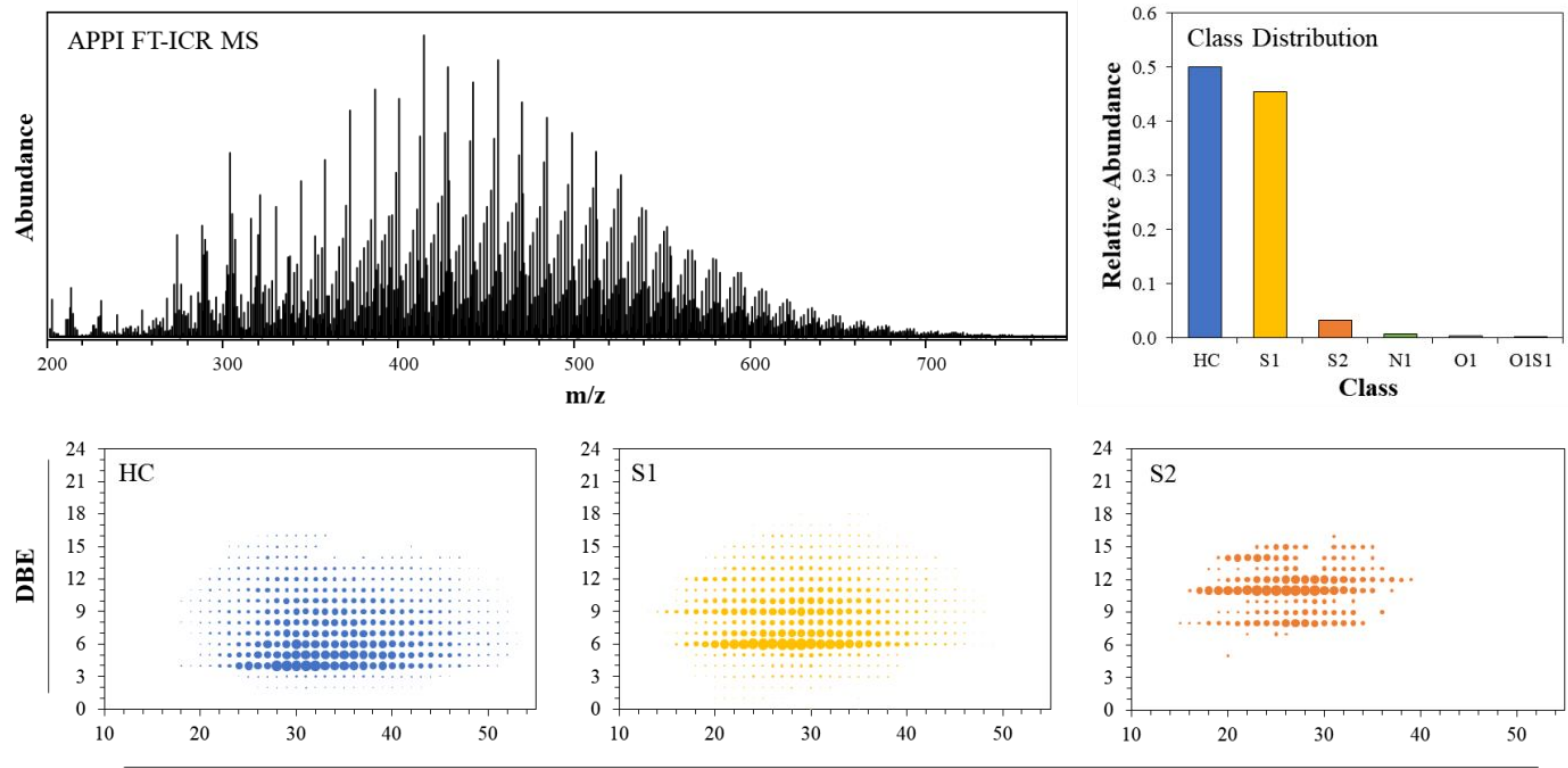

Carbon Number

b) VGO S
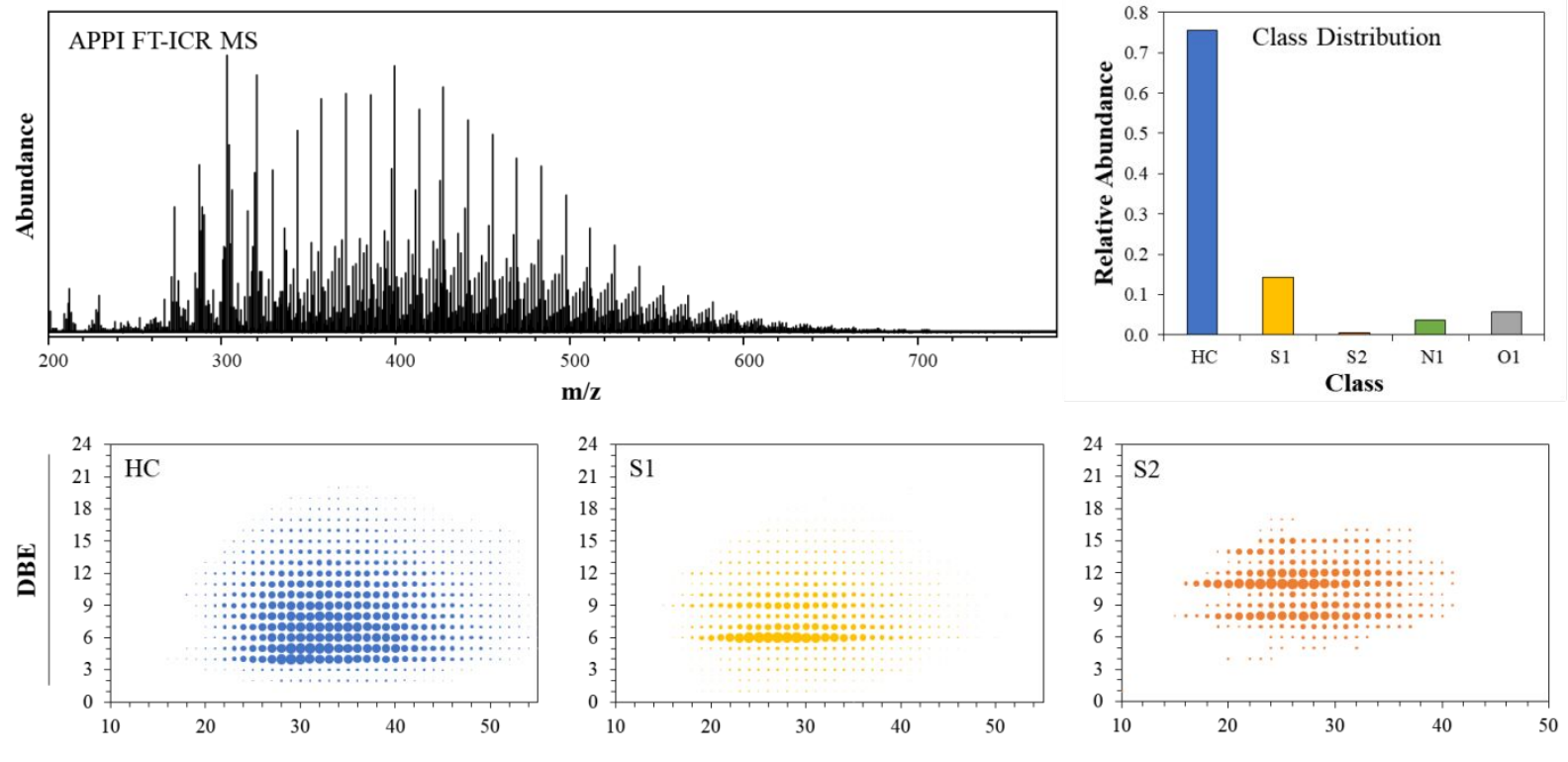

Carbon Number

Figure SI8. Mass spectrometry data for a) VGO AL and b) VGO S: APPI FT-ICR mass spectrum, class distribution, and carbon number vs DBE plots for $\mathrm{HC}, \mathrm{S} 1$, and S2 class species, respectively, where the dots' area represents their mass spectral abundance. 


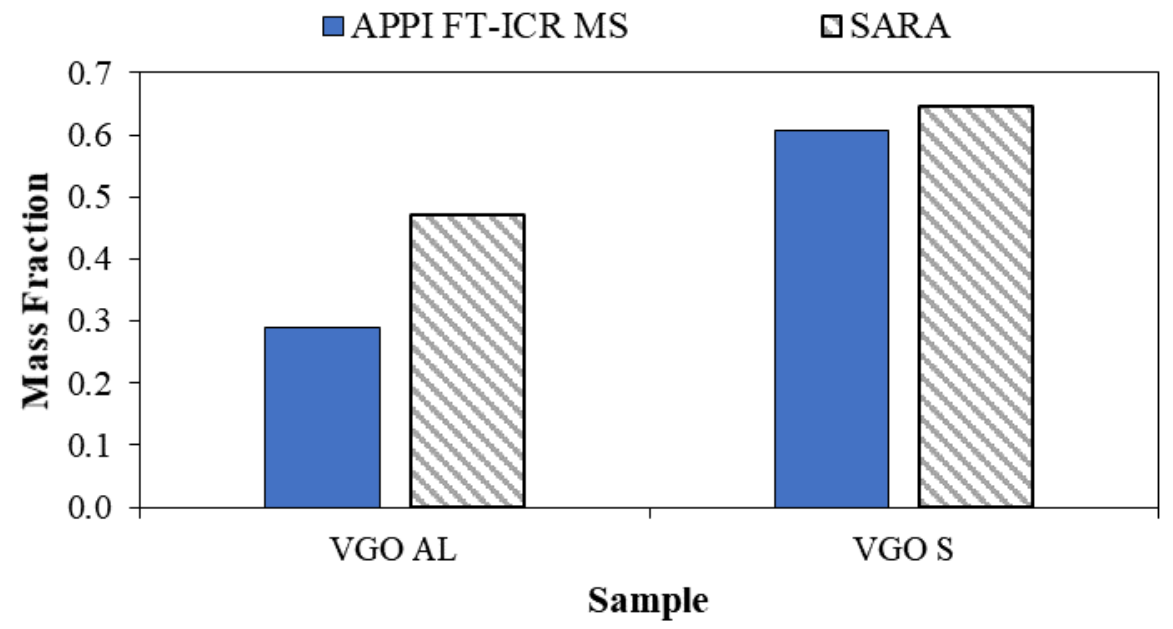

Figure SI9. Mass fraction of saturated compounds estimated by APPI FT-ICR MS and gravimetrically determined by liquid chromatograph fractionation (SARA).

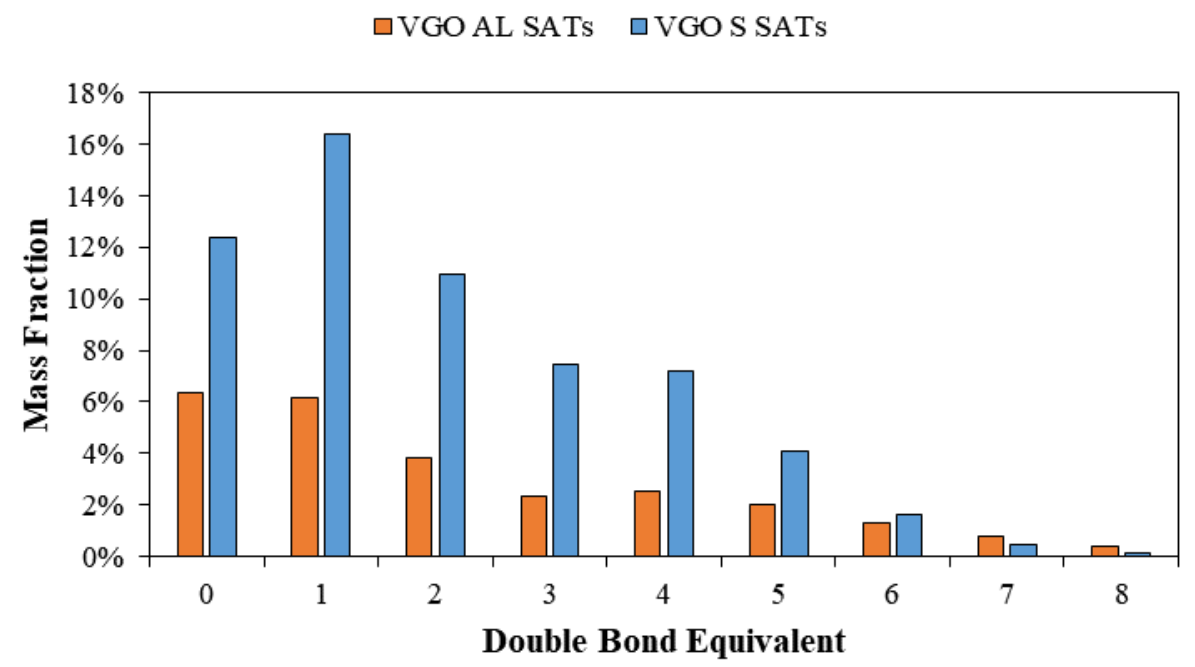

Figure SI10. DBE distribution of the VGO AL and S sample saturated compounds (SATs) fractions based on FD TOF MS analysis. For saturated compounds represented here, DBE reflects directly the number of naphthenic rings. 